\title{
Trifecta results in Retzius-sparing robotic radical prostatectomy: results of a high-volume center
}

\author{
Alberto Olivero ${ }^{1}$, Paolo Dell’Oglio ${ }^{1,2,3}$, Francesca Ambrosini ${ }^{4}$, Silvia Secco ${ }^{1}$, Michele Barbieri ${ }^{1}$, Erika \\ Palagonia $^{1,5}$, Giancarlo Napoli ${ }^{1}$, Elena Strada ${ }^{1}$, Giovanni Petralia ${ }^{1}$, Dario Di Trapani ${ }^{1}$, Carlo Buratto ${ }^{1}$, Marco \\ Martiriggiano ${ }^{4}$, Antonio Galfano ${ }^{1}$, Aldo Massimo Bocciardi ${ }^{1}$ \\ 'Department of Urology, ASST Grande Ospedale Metropolitano Niguarda, Milan 20121, Italy. \\ ${ }^{2}$ Department of Urology, Netherlands Cancer Institute-Antoni van Leeuwenhoek Hospital, Amsterdam 1066, The Netherlands. \\ ${ }^{3}$ Interventional Molecular Imaging Laboratory, Department of Radiology, Leiden University Medical Center, Leiden 2333, The \\ Netherlands \\ ${ }^{4}$ Department of Urology, Policlinico San Martino Hospital, University of Genova, Genova 16131, Italy. \\ 5Division of Urology, University Hospital "Ospedali Riuniti", Marche Polytechnic University, Ancona 60126, Italy.
}

Correspondence to: Dr. Alberto Olivero, Department of Urology, ASST Grande Ospedale Metropolitano Niguarda, piazza Ospedale Maggiore 3, Milan 20121, Italy. E-mail: oliveroalby@gmail.com

How to cite this article: Olivero A, Dell'Oglio P, Ambrosini F, Secco S, Barbieri M, Palagonia E, Napoli G, Strada E, Petralia G, Di Trapani D, Buratto C, Martiriggiano M, Galfano A, Bocciardi AM. Trifecta results in Retzius-sparing robotic radical prostatectomy: results of a high-volume center. Mini-invasive Surg 2022;6:6. https://dx.doi.org/10.20517/2574-1225.2021.117

Received: 18 Oct 2021 First Decision: 25 Nov 2021 Revised: 23 Dec 2021 Accepted: 29 Dec 2021 Published: 17 Jan 2022

Academic Editors: Giulio Belli, Riccardo Autorino, Enrico Checcucci Copy Editor: Xi-Jun Chen Production Editor: Xi-Jun Chen

\begin{abstract}
Aim: We aimed to evaluate trifecta outcomes after Retzius-sparing robot-assisted radical prostatectomy (rsRARP).

Methods: We evaluated 1488 patients who had undergone rs-RARP at our institution from 2011 to 2019 . All patients filled out questionaries for functional outcomes before surgery, and only patients with baseline continence and IIEF-5 scores of $>16$ were included. Biochemical recurrence (BCR) was defined as two consecutive prostatic specific antigen levels of $>0.2 \mathrm{ng} / \mathrm{mL}$ after rs-RARP. Postoperative continence was defined as the use of no pads. Potency was defined as the ability to achieve erections for sexual intercourse, with or without phosphodiesterase- 5 (PDE-5) inhibitors. A multivariable logistic regression model was performed to identify predictors of trifecta outcome.
\end{abstract}

Results: In total, 1240 patients were included in the analysis. During the 24-month follow-up time, 149 patients (11.9\%) harbored BCR. Urinary continence was observed in 981 patients (79.5\%), while 171 (13.8\%) still used a safety pad daily after 24 months. Sexual potency was reported in 643 patients (51.9\%), of whom 379 (30.6\%) had 
spontaneous erections and 264 (21.3\%) used a PDE-5 inhibitor. Overall, the trifecta outcome was reached by $42.1 \%$ of the study's population. The trifecta outcome was easily reached by younger patients and patients who underwent a full nerve-sparing (NS) prostatectomy. In the multivariable model, age [odds ratio $(O R)=0.89 ; 95 \%$ confidence interval $(\mathrm{Cl}): 0.84-0.90 ; P<0.01$ ] and type of NS surgery [partial NS (OR $=3.34 ; 95 \% \mathrm{Cl}: 1.01-11 ; P=$ $0.04)$ full NS (OR $=4.57 ; 95 \% \mathrm{Cl}: 1.86-12 ; P<0.01)$ ] resulted as independent predictors.

Conclusion: rs-RARP is associated with optimal trifecta outcome rate. Age and NS technique are independent predictors of trifecta outcomes.

Keywords: Prostate cancer, robot-assisted radical prostatectomy, Retzius sparing, functional outcomes, trifecta outcome

\section{INTRODUCTION}

Robot-assisted radical prostatectomy (RARP) was introduced in the early $2000 \mathrm{~s}^{[1]}$. Since its introduction, RARP has gained popularity among surgeons due to the well-known advantages, such as three-dimensional binocular magnification, motion scaling, tremor filtration, and good surgeon ergonomics ${ }^{[2]}$. Furthermore, since radical treatment of prostate cancer $(\mathrm{PCa})$ is associated with optimal oncologic outcomes ${ }^{[3]}$, the focus of the urologist is to improve the quality of life (QoL) after treatment, and urinary continence and sexual potency preservation are the two most frequent QoL alterations that bother patients after RARP ${ }^{[4]}$.

The three main long-term goals of RARP are complete removal of cancer, recovery of continence, and recovery of potency, which are commonly gathered with the name of "trifecta outcome" ${ }^{[5]}$. Various studies have reported the rate of trifecta outcome in RARP ${ }^{[6,7]}$, ranging from $23 \%$ to $86 \%$. This considerable variability is mainly due to the different definitions of trifecta adopted in the literature ${ }^{[8]}$.

Retzius-sparing robot-assisted radical prostatectomy (rs-RARP) was first proposed by our group in $2010^{[9]}$ and is recognized by the European Association of Urology (EAU) guidelines as an effective surgical option for PCa treatment ${ }^{[10]}$. The technique's success is due to a shorter continence recovery time than the traditional robotic approach ${ }^{[11-13]}$. Recently, it has been demonstrated that the technique is also safe during the learning curve phase ${ }^{[13-15]}$.

Although urinary continence recovery and other specific outcomes after rs-RARP have been described ${ }^{[16,17]}$, no series analyzing the trifecta rates extensively after rs-RARP have been previously published, and further research is needed to evaluate both sexual function outcomes and oncologic outcomes ${ }^{[18]}$.

This study aimed to evaluate trifecta outcome rates at 24 months after rs-RARP and identify possible outcomes predictors.

\section{METHODS}

\section{Study population}

Data from 1488 PCa patients treated with rs-RARP at a single high-volume European center (ASST Grande Ospedale Metropolitano Niguarda, Milan, Italy) between January 2011 and March 2019 were analyzed. Data were extrapolated from an ongoing prospective database, and all patients had at least 24 months of followup. 
All patients prior to the surgery answered self-administered validated questionnaire, the International Index of Erectile Function (IIEF-5) ${ }^{[19]}$, to assess the baseline sexual function.

In the present study, we only included patients with preoperative IIEF- 5 scores of $>16$. In addition, all patients analyzed were continent before rs-RARP. All patients provided informed consent.

\section{Surgical technique and functional rehabilitation}

All RS-RARP procedures were performed with a four-arm da Vinci Si Surgical System (Intuitive Surgical, Sunnyvale, CA, USA) with a transperitoneal approach. Eight different urologists with different grades of experience performed the surgeries. All the surgeons had completed the learning curve and had completed at least 40 cases. The assistant was often a resident or a urologist in the learning curve phase. Patients were placed in the $30^{\circ}$ Trendelenburg position. Six laparoscopic trocars were used as previously described ${ }^{[9]}$. The type of nerve sparing (NS) performed during the rs-RARP was reported according to the Pasadena Consensus Panel ${ }^{[20]}$. In the surgical report, the surgeon defined a grade of NS for each side of the gland (intra-fascial, inter-fascial, or extra-fascial/no nerve-sparing technique). We considered full NS intra-fascial at least on one side and intra- or inter-fascial on the other, partial NS inter-fascial bilaterally, and minimal or no NS for extra-fascial or no NS on one side. Therefore, all types of NS were included in the analysis.

All patients started pelvic floor muscle exercises immediately after the supra-pubic catheter removal and were recommended to continue during the follow-up. In addition, all patients were advised to use phosphodiesterase-5 (PDE-5) inhibitors after surgery regularly, at least three times/week, until recovery of sexual function. A vacuum device was not routinely recommended.

\section{Follow-up and outcomes definition}

Our primary goal was to determine the trifecta rate 24 months after rs-RARP.

Urinary continence was defined as the use of no pads (score 0) in the last four weeks.

Potency was defined as the ability to obtain and maintain a proper erection for sexual intercourse in more than $50 \%$ of the attempts, with or without PDE-5 inhibitors.

The biochemical recurrence (BCR) was defined as two consecutive prostatic specific antigen (PSA) measurements of $>0.2 \mathrm{ng} / \mathrm{mL}$ after the surgery.

During follow-up, serum PSA measurement and clinical evaluation were carried out at 3, 6, and 12 months, and then every six months for three years. Before clinical evaluations, questions about potency and urinary function were assessed.

\section{Statistical analyses}

Medians and interquartile ranges (IQR) and frequencies and proportions were reported for continuous and categorical variables, respectively.

To identify predictors of trifecta outcome, a multivariable logistic regression model testing for trifecta outcome was performed. Adjustment variables consisted of age, body max index (BMI), PSA, comorbidities (reported as the Charlson score), prostate volume, pathological International Society of Urological Pathology (ISUP) grade, pathological tumor stage (pT), and nerve-sparing technique. The R software environment for statistical computing and graphics (version 3.6.3) was used for all statistical analyses. All 
tests were two-sided with a level of significance set at $P<0.05$.

\section{RESULTS}

Of the 1488 patients treated, 167 were excluded due to a preoperatory IEEF-5 score of $<17$ and 81 were excluded because of incomplete data or follow-up. Thus, in total, 1240 patients were included in the analysis.

\section{Preoperative characteristics}

Table 1 summarizes the descriptive characteristics of our patients. Median age and BMI were 65 years (IQR: 60-69 years) and $26.1 \mathrm{~kg} / \mathrm{m}^{2}$ (IQR: 24.2-28.4 kg/m²). Median PSA was $6.9 \mathrm{ng} / \mathrm{mL}$ (IQR: 5-9.9 ng/mL). Most of the patients presented a clinical T2a or inferior disease (56.5\%). ISUP grade group was 1 in $582(46.4 \%), 2$ in $322(25.8 \%), 3$ in $133(10.5 \%), 4$ in $152(12.2 \%)$, and 5 in $51(4.1 \%)$ of the patients. The median prostate volume was $42 \mathrm{~mL}$ (IQR: $32-55 \mathrm{~mL}$ ).

Overall, $71.7 \%$ of the patients underwent a full NS procedure, $4.1 \%$ underwent a partial NS surgery, and $24.2 \%$ underwent a minimal or non-NS prostatectomy.

\section{Functional and cancer control outcomes}

Positive surgical margins were present in 215 patients (17.3\%). During the 24-month follow-up period, 149 patients (11.9\%) harbored BCR.

Urinary continence was observed in 981 patients (79.5\%), while $171(13.8 \%)$ still used a safety pad daily after 24 months. In total, 88 patients $(6.7 \%)$ did not reach urinary continence.

Sexual potency at 24 months was reported in 643 patients (51.9\%), of whom 379 (30.6\%) had spontaneous erections and $264(21.3 \%)$ used PDE-5 inhibitor. Overall, 34 patients $(2.7 \%)$ were able to reach erections with intracavernous injections, while the remaining $563(45.4 \%)$ patients did not reach a satisfactory erection for intercourse. The full results are shown in Table 2.

\section{Trifecta outcomes}

Overall, the trifecta outcome was reached by $42.1 \%$ of the study's population.

Trifecta rate stratified for the grade of NS surgery was $20.7 \%$ for minimal or no NS, $38.8 \%$ for partial NS, and $49.9 \%$ for full NS [Figure $1 \mathrm{~A}$ and $\mathrm{B}$ ].

Trifecta outcome rate stratified for age groups was as follows: $62.2 \%$ in patients younger than 56 years, $58.2 \%$ inpatient between 56 and 60 years, $45.8 \%$ in patients between 61 and 65 years, $34.2 \%$ in patients between 66 and 70 years, $26.1 \%$ in patients between 71 and 75 years, and $9.1 \%$ in patients older than 75 years [Figure $2 \mathrm{~A}$ and $\mathrm{B}$ ].

A multivariable logistic regression analysis predicting trifecta found that age (OR $=0.89 ; 95 \% \mathrm{CI}$ : 0.84-0.90; $P$ $<0.01)$ and the grade of nerve-sparing surgery of partial NS (OR $=3.34 ; 95 \% \mathrm{CI}: 1.01-11 ; P=0.04) v$ s. full NS $(\mathrm{OR}=4.57 ; 95 \% \mathrm{CI}: 1.86-12 ; P<0.01)$ reached independent predictor status [Table 3].

\section{DISCUSSION}

High-level evidence evaluating the trifecta outcome rate after rs-RARP is unavailable. Most studies have evaluated the intraoperative outcomes and urinary continence results after rs-RARP ${ }^{[13]}$. To improve the 
Table 1. Descriptive characteristics of $\mathbf{1 2 4 0}$ prostate cancer patients treated with the Retzius-sparing approach at a single European high-volume center

\begin{tabular}{|c|c|}
\hline \multicolumn{2}{|l|}{ Preoperative variables $(n=1240)$} \\
\hline Age, years, median (IQR) & $65(60-69.3)$ \\
\hline BMI, kg/mq, median (IQR) & $26.1(24.2-28.4)$ \\
\hline $\begin{array}{l}\text { Charlson comorbidity index, } n(\%) \\
0 \\
1 \\
2 \\
3\end{array}$ & $\begin{array}{l}919(74.1) \\
141(11.4) \\
120(9.7) \\
60(4.8)\end{array}$ \\
\hline Previous abdominal surgery, $n(\%)$ & 409 (33) \\
\hline Previous surgery for $\mathbf{B P H}, n(\%)$ & $57(4.6)$ \\
\hline PSA at RS-RARP, ng/mL, median (IQR) & $6.9(5-9.9)$ \\
\hline $\begin{array}{l}\text { Clinical tumour stage, } n(\%) \\
\leq c T 2 a \\
\text { cT2b } \\
\text { cT2c } \\
\geq \text { cT3 }\end{array}$ & $\begin{array}{l}703(56.5) \\
305(24.6) \\
146(11.8) \\
88(7.1)\end{array}$ \\
\hline $\begin{array}{l}\text { ISUP grade group at prostate biopsy, } n \text { ( } \\
1 \\
2 \\
3 \\
4 \\
5\end{array}$ & $\begin{array}{l}582(46.4) \\
322(25.8) \\
133(10.5) \\
152(12.2) \\
51(4.1)\end{array}$ \\
\hline $\begin{array}{l}\text { EAU risk classification group, } n(\%) \\
\text { Low } \\
\text { Intermediate } \\
\text { High }\end{array}$ & $\begin{array}{l}412(33.2) \\
525(42.4) \\
303(24.4)\end{array}$ \\
\hline Prostate volume, mL, median (IQR) & $42(32-55)$ \\
\hline Intraoperative variables $(n=1240)$ & \\
\hline $\begin{array}{l}\text { Nerve sparing technique, } n(\%) \\
\text { Full NS } \\
\text { Partial NS } \\
\text { Non NS }\end{array}$ & $\begin{array}{l}891(71.7) \\
49(4.1) \\
300(24.2)\end{array}$ \\
\hline
\end{tabular}

BMI: Body mass index; BPH: benign prostate hyperplasia; PSA: prostate specific antigen; ISUP: International Society of Urological Pathology; RSRARP: Retzius-sparing robot-assisted radical prostatectomy; IQR: inter-quartile range; NS: nerve sparing; ISUP: International Society of Urological Pathology; EAU: European Association of Urology.

counseling of PCa patients and better inform them regarding what to expect after the surgery, we analyzed the trifecta outcome at 24 months after the surgery. We investigated the possible predictor factors in over 1200 patients who had undergone rs-RARP in a high-volume center. We found that more than $40 \%$ of the patients 24 months after rs-RARP reached the trifecta outcome, regardless of the patients' age or the NSapproach used during the surgery.

Moreover, we found that age and NS technique are independent predictors of the trifecta outcome. Lastly, as previously demonstrated, we observed that the potency rate is the item that most influences the trifecta rate as only slightly more than $50 \%$ of our group was potent after rs-RARP.

Our findings will help better assess the risk of not reaching the trifecta outcome after rs-RARP and give valuable information for the preoperatory counseling of PCa patients. 
Table 2. Cancer control and functional outcomes of 1240 prostate cancer patients treated with the Retzius-sparing approach at a single European high-volume center

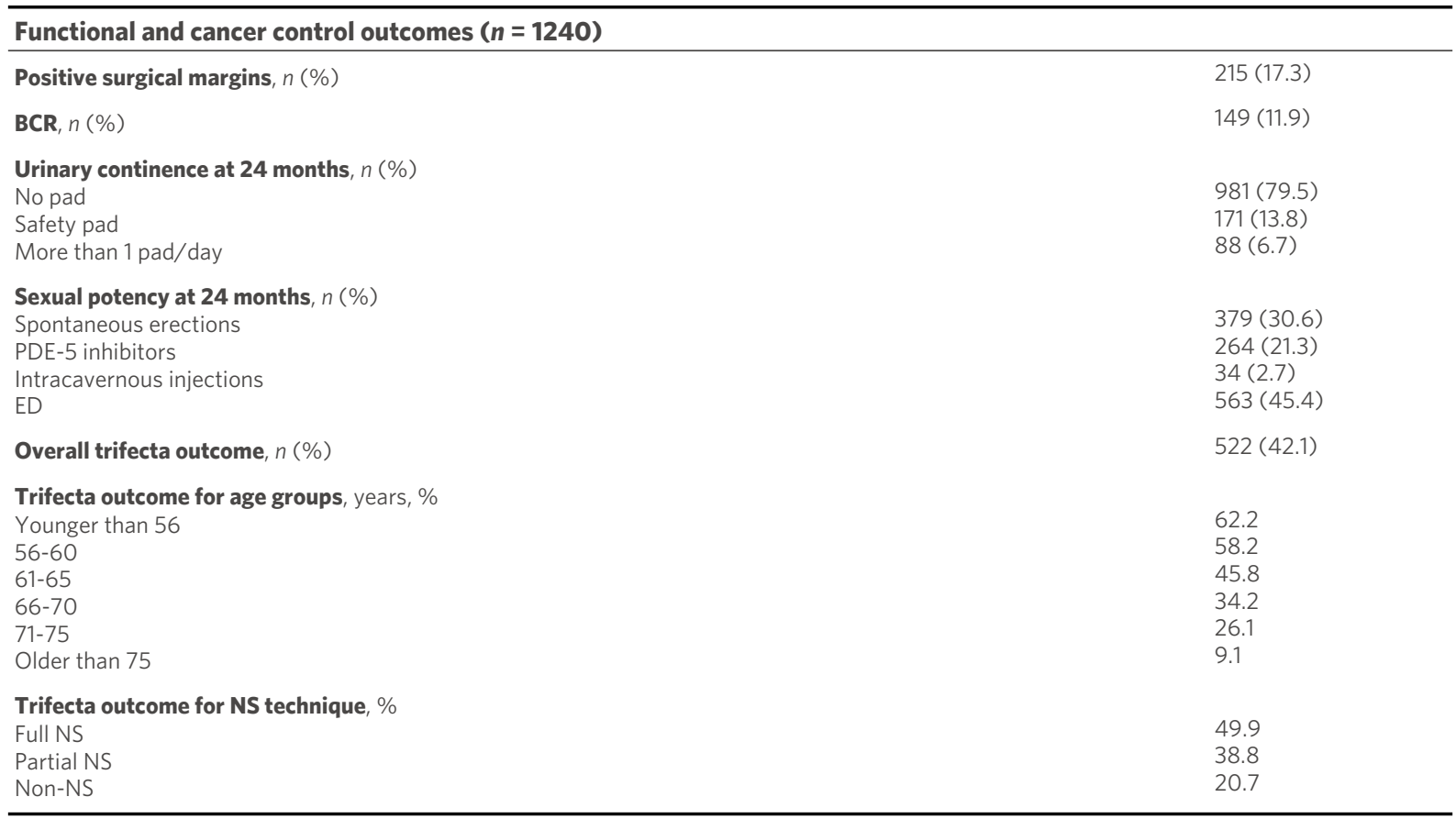

BCR: Biochemical recurrence; ED: erectile disfunction; NS: nerve sparing.

Table 3. Multivariable logistic regression model predicting trifecta in 1240 prostate cancer patients treated with the Retzius-sparing approach at a single European high-volume center

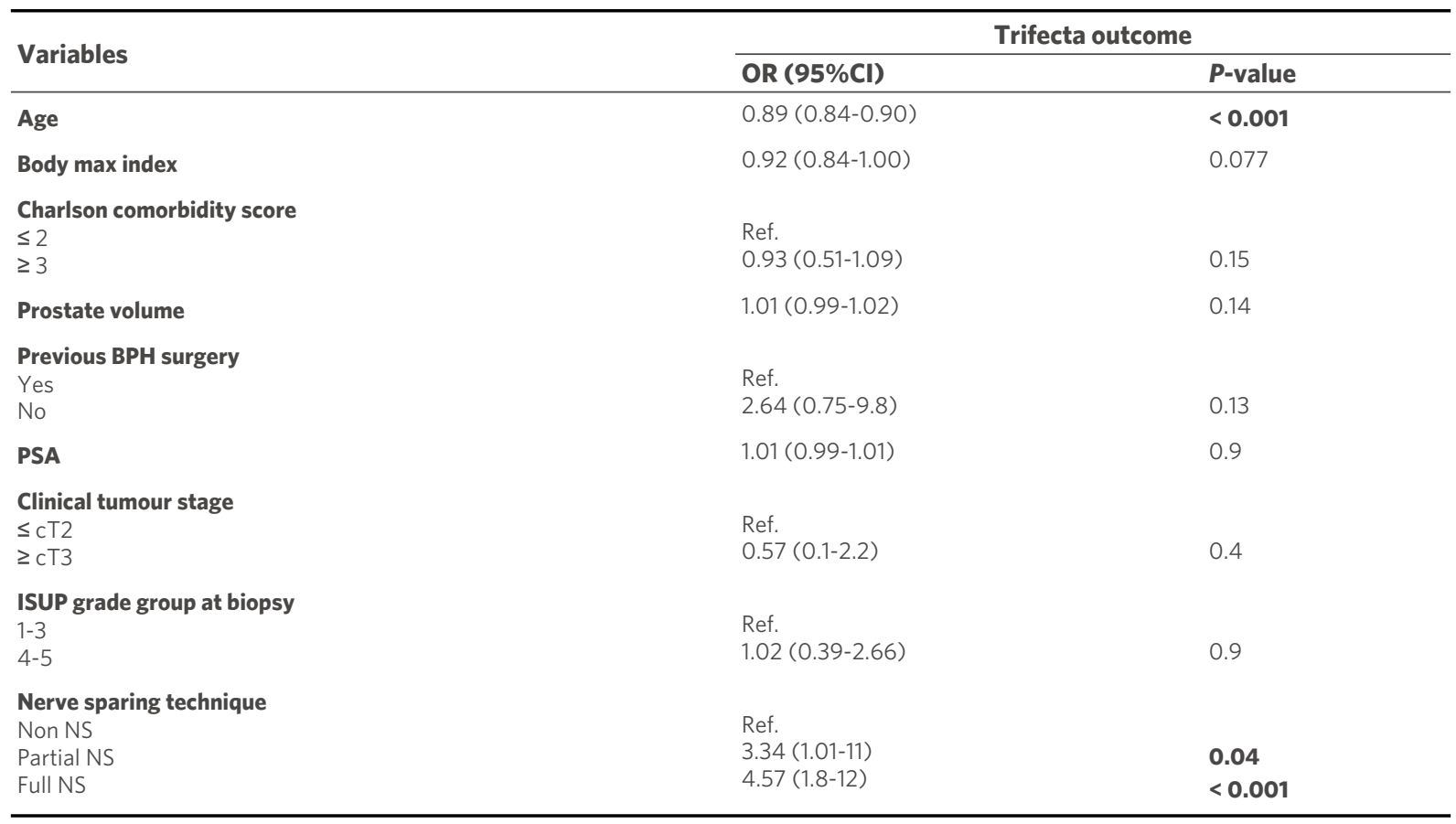

OR: Odds ratio; $\mathrm{Cl}$ : confidence interval. 

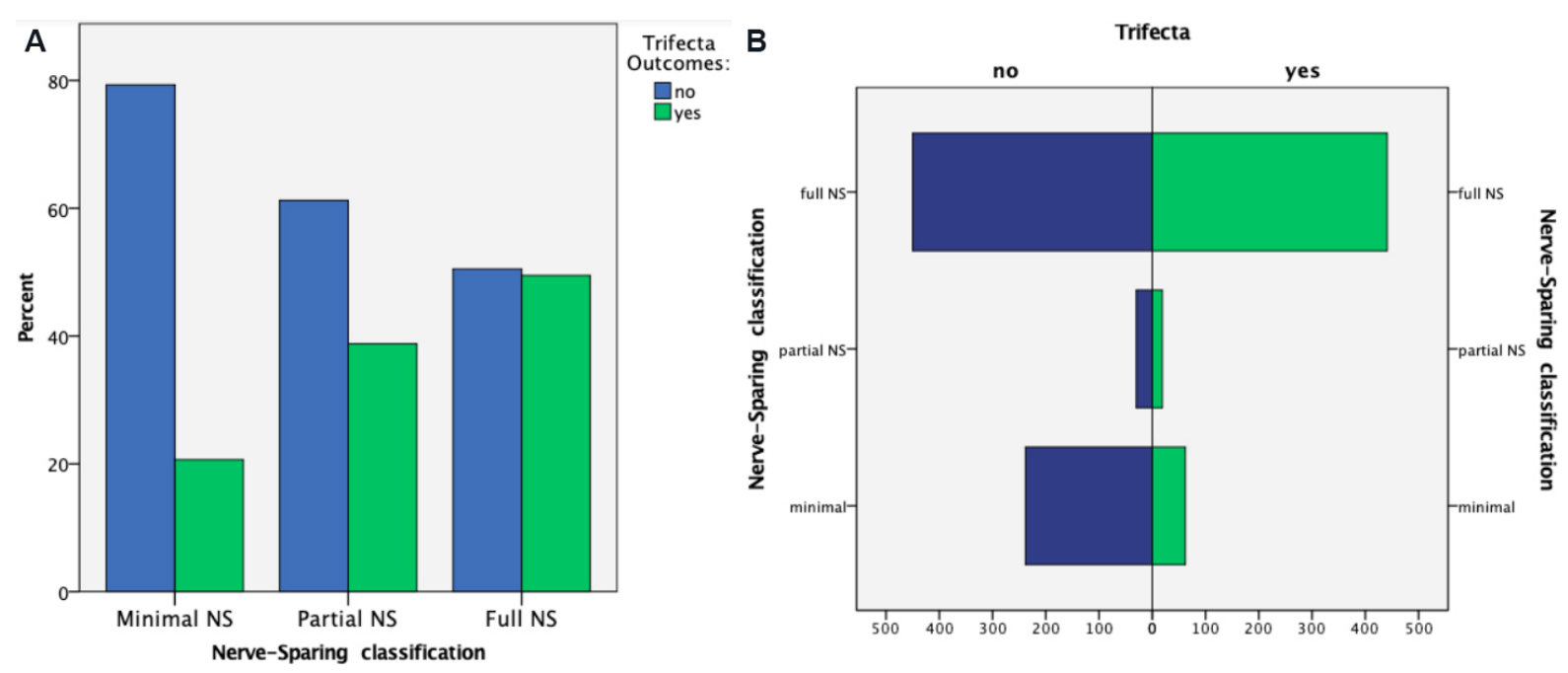

Figure 1. Histogram of distribution of Trifecta outcome rate stratified by NS-approach groups: (A) percentage; and (B) count. NS: Nerve sparing.
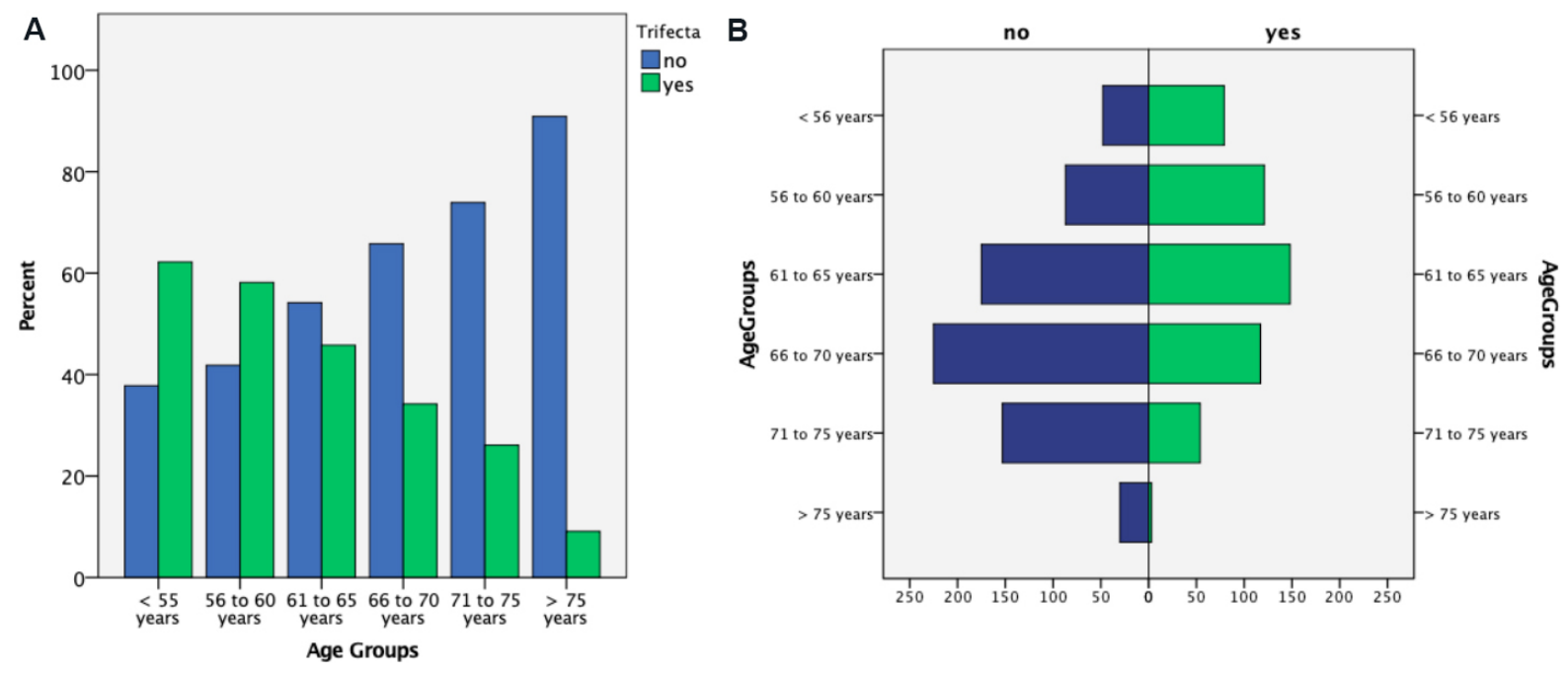

Figure 2. Histogram of distribution of Trifecta outcome rate stratified by age groups: (A) percentage; and (B) count.

Modern PCa surgery aims to achieve cancer control while minimizing its impact on patients' QoL $^{[21]}$. Since the introduction of robot-assisted radical prostatectomy, there has been constant research on technical improvements and technique refinements to improve functional outcomes ${ }^{[22-24]}$. Trifecta outcome is considered a valuable tool to summarize the result of radical prostatectom ${ }^{[5]}$, and, since its introduction, various series reported rates of achieving the outcome ranging from $23 \%$ to $86^{[2,6,7,25]}$. The vast range reported is a direct consequence of the heterogenicity adopted in defining the single outcomes. While the definition of BCR as two consecutive PSA values of $\geq 0.2 \mathrm{ng} / \mathrm{mL}$ is standardized in the literature, the definitions proposed for urinary continence and sexual function are various ${ }^{[8]}$. Several authors used "no leak" to define continence ${ }^{[7]}$, while others classified continence according to the number of pads used daily ${ }^{[6]}$. The use of a single daily safety pad was often debated as an achievement of urinary continence and has been adopted as a definition in several studies ${ }^{[2,27]}$. In our study, we chose to use only completely dry patients to fulfill patients' expectations; this led us to reduce our continence rate from $93.3 \%$ to $79.5 \%$. 
Several definitions have been proposed to evaluate erectile function after surgery: partial recovery, adequate rigidity, the ability for intercourse, and sexual satisfaction. Some authors also expanded the definition from couple sexual intercourse to sexual activity, including masturbation ${ }^{[4]}$. We used the most used definition as "erection sufficient for intercourse with or without the use of a PDE-5"

Another substantial difference of our work from the literature is that we included all patients submitted to rs-RARP without selecting for NS-technique or age. As shown in the figures, trifecta outcome resulted inversely proportional to patient's age and correlated to the grade of NS adopted; ideally, in a selected population of young patients treated with full NS, we can report trifecta rates around $66 \%$ in accordance with the best results published.

A recent systematic review found a role in the recovery of continence and consequently in the trifecta rate for the various anatomical reconstructions after RARP, finding that anterior and posterior reconstructions together facilitate continence recovery in comparison to only one approach ${ }^{[28]}$.

Our study is the first to investigate trifecta outcome after rs-RARP extensively. We relied on a significant number of patients and sufficient follow-up time. In addition, we overcame the potential bias of the single operator ability by including different surgeons.

Another strong point of this study is that other authors included very few high-risk prostate cancer cases $^{[6,7,26]}$. In our group, more than $24 \%$ of patients were classified high risk according to EAU risk group. These are the patients who historically do not reach the trifecta outcome, and such a rate could limit the result in our cohort.

Our study must be interpreted considering some limitations. First, our findings derived from a retrospective review of prospectively collected observational data. Thus, our results must be interpreted considering the limitations of such data. Second, the follow-up time of 24 months is relatively short, and only early intermediate oncological results can be deducted.

Third, this study reports single-center experience where rs-RARP was ideated. In our center, only the posterior approach is performed; this may not be translated to other centers where surgeons are not so experienced in the Retzius-sparing approach. Confirmation studies in other centers and confrontation studies between rs-RARP and anterior approach may be needed to obtain Level 1 evidence.

In conclusions, rs-RARP is associated with promising trifecta outcome rate. Age and NS technique are independent predictors of trifecta outcomes. The Retzius-sparing approach should be considered a valid surgical treatment for all PCa patients who want to optimize the balance between oncological and functional outcomes.

\section{DECLARATIONS}

\section{Authors' contributions}

Data analysis and manuscript drafting: Olivero A, Dell'Oglio P, Ambrosini F

Data research technical, and material support: Barbieri M, Palagonia E, Napoli G, Di Trapani D, Buratto C, Martiriggiano M

Manuscript revision: Bocciardi AM, Galfano A, Petralia G, Strada E, Secco S 


\section{Availability of data and materials}

Not applicable.

\section{Financial support and sponsorship}

None.

\section{Conflicts of interest}

All authors declared that there are no conflicts of interest.

\section{Ethical approval and consent to participate}

The study has been designed and performed in accordance with the Declaration of Helsinki. An informed consent to utilize the data for research purposes was obtained from all participants.

\section{Consent for publication}

Not applicable.

\section{Copyright}

(c) The Author(s) 2022.

\section{REFERENCES}

1. Sood A, Abdollah F, Menon M. Retzius-sparing robot-assisted radical prostatectomy. BJU Int 2019;123:7-8. DOI PubMed

2. Patel VR, Abdul-Muhsin HM, Schatloff O, et al. Critical review of 'pentafecta' outcomes after robot-assisted laparoscopic prostatectomy in high-volume centres. BJU Int 2011;108:1007-17. DOI PubMed

3. Bianchi L, Gandaglia G, Fossati N, et al. Oncologic outcomes in prostate cancer patients treated with robot-assisted radical prostatectomy: results from a single institution series with more than 10 years follow up. Minerva Urol Nefrol 2019;71:38-46. DOI PubMed

4. Inoue S, Hieda K, Hayashi T, Teishima J, Matsubara A. Longitudinal analysis of trifecta outcome in Japanese patients with prostate cancer following robot-assisted laparoscopic radical prostatectomy. World J Urol 2020. DOI PubMed

5. Bianco FJ Jr, Scardino PT, Eastham JA. Radical prostatectomy: long-term cancer control and recovery of sexual and urinary function ("trifecta"). Urology 2005;66:83-94. DOI PubMed

6. Patel VR, Coelho RF, Chauhan S, et al. Continence, potency and oncological outcomes after robotic-assisted radical prostatectomy: early trifecta results of a high-volume surgeon. BJU Int 2010;106:696-702. DOI PubMed

7. Novara G, Ficarra V, D'Elia C, Secco S, Cavalleri S, Artibani W. Trifecta outcomes after robot-assisted laparoscopic radical prostatectomy. BJU Int 2011;107:100-4. DOI PubMed

8. Borregales LD, Berg WT, Tal O, et al. 'Trifecta' after radical prostatectomy: is there a standard definition? BJU Int 2013;112:60-7. DOI PubMed

9. Galfano A, Ascione A, Grimaldi S, Petralia G, Strada E, Bocciardi AM. A new anatomic approach for robot-assisted laparoscopic prostatectomy: a feasibility study for completely intrafascial surgery. Eur Urol 2010;58:457-61. DOI PubMed

10. Mottet N, van den Bergh RCN, Briers E, et al. EAU-EANM-ESTRO-ESUR-SIOG Guidelines on Prostate Cancer-2020 update. Part 1: screening, diagnosis, and local treatment with curative intent. Eur Urol 2021;79:243-62. DOI PubMed

11. Dalela D, Jeong W, Prasad MA, et al. A pragmatic randomized controlled trial examining the impact of the Retzius-sparing approach on early urinary continence recovery after robot-assisted radical prostatectomy. Eur Urol 2017;72:677-85. DOI PubMed

12. Menon M, Dalela D, Jamil M, et al. Functional recovery, oncologic outcomes and postoperative complications after robot-assisted radical prostatectomy: an evidence-based analysis comparing the Retzius sparing and standard approaches. J Urol 2018;199:1210-7. DOI PubMed

13. Checcucci E, Veccia A, Fiori C, et al. Retzius-sparing robot-assisted radical prostatectomy vs the standard approach: a systematic review and analysis of comparative outcomes. BJU Int 2020;125:8-16. DOI PubMed

14. Olivero A, Galfano A, Piccinelli M, et al. Retzius-sparing robotic radical prostatectomy for surgeons in the learning curve: a propensity score-matching analysis. Eur Urol Focus 2021;7:772-8. DOI PubMed

15. Galfano A, Secco S, Bocciardi AM, Mottrie A. Retzius-sparing robot-assisted laparoscopic radical prostatectomy: an international survey on surgical details and worldwide diffusion. Eur Urol Focus 2020;6:1021-3. DOI PubMed

16. Galfano A, Secco S, Panarello D, et al. Pain and discomfort after Retzius-sparing robot-assisted radical prostatectomy: a comparative study between suprapubic cystostomy and urethral catheter as urinary drainage. Minerva Urol Nefrol 2019;71:381-5. DOI PubMed

17. Kowalczyk KJ, Davis M, O'Neill J, et al. Impact of Retzius-sparing versus standard robotic-assisted radical prostatectomy on penile shortening, Peyronie's disease, and inguinal hernia sequelae. Eur Urol Open Sci 2020;22:17-22. DOI PubMed PMC

18. Davis M, Egan J, Marhamati S, Galfano A, Kowalczyk KJ. Retzius-sparing robot-assisted robotic prostatectomy: past, present, and future. Urol Clin North Am 2021;48:11-23. DOI PubMed 
19. Rosen RC, Cappelleri JC, Smith MD, Lipsky J, Peña BM. Development and evaluation of an abridged, 5-item version of the International Index of Erectile Function (IIEF-5) as a diagnostic tool for erectile dysfunction. Int J Impot Res 1999;11:319-26. DOI PubMed

20. Montorsi F, Wilson TG, Rosen RC, et al; Pasadena Consensus Panel. Best practices in robot-assisted radical prostatectomy: recommendations of the Pasadena Consensus Panel. Eur Urol 2012;62:368-81. DOI PubMed

21. Autorino R, Porpiglia F, Dasgupta P, et al. Precision surgery and genitourinary cancers. Eur J Surg Oncol 2017;43:893-908. DOI PubMed

22. Porpiglia F, Bertolo R, Manfredi M, et al. Total anatomical reconstruction during robot-assisted radical prostatectomy: implications on early recovery of urinary continence. Eur Urol 2016;69:485-95. DOI PubMed

23. Puliatti S, Elsherbiny A, Eissa A, et al. Effect of puboprostatic ligament reconstruction on continence recovery after robot-assisted laparoscopic prostatectomy: our initial experience. Minerva Urol Nefrol 2019;71:230-9. DOI PubMed

24. Campobasso D, Fiori C, Amparore D, et al. Total anatomical reconstruction during robot-assisted radical prostatectomy in patients with previous prostate surgery. Minerva Urol Nefrol 2019;71:605-11. DOI PubMed

25. Xylinas E, Ploussard G, Durand X, et al. Evaluation of combined oncological and functional outcomes after radical prostatectomy: trifecta rate of achieving continence, potency and cancer control--a literature review. Urology 2010;76:1194-8. DOI PubMed

26. Eastham JA, Scardino PT, Kattan MW. Predicting an optimal outcome after radical prostatectomy: the trifecta nomogram. J Urol 2008;179:2207-10; discussion 2210-1. DOI PubMed PMC

27. Shikanov SA, Zorn KC, Zagaja GP, Shalhav AL. Trifecta outcomes after robotic-assisted laparoscopic prostatectomy. Urology 2009;74:619-23. DOI PubMed

28. Checcucci E, Pecoraro A, DE Cillis S, et al; San Luigi Study Group. The importance of anatomical reconstruction for continence recovery after robot assisted radical prostatectomy: a systematic review and pooled analysis from referral centers. Minerva Urol Nephrol 2021;73:165-77. DOI PubMed 